presence of intron 22 inversion in the patients with severe hemophilia A.

Results Patients were aged 2-30 years. Six(24\%) patients were diagnosed during the neonatal period, 11(44\%) between 1-12 months, $5(20 \%)$ between $1-2$ years, $2(8 \%)$ between $2-3$ years and $1(4 \%)$ at 3.5 years. In our patients, $1-8$ joints were affected. The mean joints involvements were $3.3 \pm 1.8$. Of the 25 patients with severe hemophilia A, 7 patients had the intron 22 inversion. Seven of 15 mothers also presented with the intron 22 inversion. In all 7 cases, mother and son had the same intron 22 inversion, no new mutation was found in our patients.

Conclusion The prevalence of the intron 22 inversion in hemophilic patients is $28 \%$. This prevalence is lower than that reported Worldwide. Based on this study and other reports, we recommended that the detection of intron 22 inversion is performed as a genetic screening test in hemophilic patients.

\section{HUMAN IMMUNOGLOBULINE ROLE IN TREATMENT OF IDIOPATHIC THROMBOCYTOPENIC PURPURA}

doi:10.1136/archdischild-2012-302724.0749

'V Grajqevci, 2R Macastena, 'B Abrashi, ${ }^{2}$ F Selimi, ${ }^{2}$ E Bajrami. 'Hemato-Oncology Department; ${ }^{2}$ Pediatric Clinic, University Clinical Centre of Kosovo, Prishtina, Kosovo

Introduction Idiopathic thrombocytopenic purpura is a disorder, in which immune system destroys platelets, which are necessary for blood clotting. ITP persons have a very low platelet count. ITP appears when the immune system products antibodies against platelets. This disorder attacks equally boys and girls.

Objective The purpose of study was to present cases and treatment of the patients who are hospitalized in Pediatric Clinic in Prishtina at the hematology-Oncology unit.

Materials and Methods In this study there are included 24 cases that are hospitalized in chemato - Oncology unit during 2011 and their treatment. The diagnose is made based on anamnesis, clinical examinations, laboratory checks, biochemistry, ultrasound examinations, and bone marrow biopsy.

Results 7 cases $(29.1 \%)$ were treated with human immunoglobulin, while 17 cases (69.9\%) were treated with steroids. Immunoglobulin treatment last for 5 days. The second day of treatment with human immunoglobulin the average platelet count increase was $30 \%$ higher, while in the fifth day platelet count arrived normal values. In patient treated with steroids platelet count began to rise after one week of treatment., in most cases platelet coun was normal after two weeks of treatment.

Conclusion Immunoglobulin therapy is a very efficient therapy in acute idiopathic thrombocytopenia especially in serious and possibly fatal complications such as gastrointestinal and intracranial bleeding.

\section{DETERMINING THE MEAN CORD BLOOD IMMATURE PLATELET FRACTION (IPF) OF HEALTHY NEWBORNS}

doi:10.1136/archdischild-2012-302724.0750

'A Günlemez, ' $\mathrm{M}$ Oruç, ${ }^{2} \mathrm{HM}$ Kır, ${ }^{2} \mathrm{M}$ Çekmen, 'AS Gökalp. 'Neonataology; ' ${ }^{2}$ Biochemistry, Kocaeli University, Kocaeli, Turkey

Thrombocytopenia is the most common hematologic abnormality in newborn infants Immature platelet fraction (IPF) shows megakaryopoetic activity. The purpose of this study is to provide noninvasive new approach to thrombocytopenic infants for further studies by determining the normal levels of the mean cord blood IPF of healthy newborns.

Methods Healthy newborns who were born at Kocaeli University in 2012, took place in this study. One $\mathrm{ml}$ of cord blood was obtained into EDTA tubes. Platelet counts and the mean IPF levels were studiedstudied with XE-100 (Sysmex) device. If taking blood to detect blood group is needed in 24-48 hours, IPF levels have been reworked.

Results A total of 75 infants were enrolled in this study. Mean gestational age 37.5 , birth weight $3032 \mathrm{~g}$, platelet count $234.000 / \mathrm{mm} 3$, average levels of cord blood IPF 5.19\%, IPF level in 48th hour were found to be $4.3 \%$.

Discussion In healthy adults, the normal values of IPF has been reported as $3.4 \%$ on average from 1.1 to $6.1 \%$. Increased levels of IPF are shown to be related to increased platelet production; particularly in disorders related to the destruction of platelets and normal and low values of IPF are shown to be related to decreased platelet production conditions. There are limited number of researches which investigate IPF values in neonates.

Conclusion In this study we found average levels of cord blood is IPF $5.19 \%$. Determining the right approach to thrombocytopenic patients will be possible by recognizing the normal ranges of IPF values in healthy newborns.

\section{ARE INFANTS OF DIABETIC MOTHERS MORE PRONE TO IRON DEFICIENCY?}

doi:10.1136/archdischild-2012-302724.0751

${ }^{1} S \mathrm{C}$ Coşkun, 'H Bilgen, 'H Özdemir, ${ }^{2} \mathrm{O}$ Şirikçi, ${ }^{1} \mathrm{E}$ Özek. ${ }^{1}$ Marmara University School of Medicine Department of Pediatrics, Neonatology; 'Marmara University School of Medicine, Department of Biochemistry, Istanbul, Turkey

Background Iron deficiency during the fetal and neonatal (perinatal) period can result in dysfunction of multiple organ systems, some of which might not recover despite iron rehabilitation. The aim of the study was to assess whether infants born to mothers with gestational diabetes (GDMs)and large for gestational age (LGA) infants are at higher risk for iron-deficiency compared to matched healthy term controls.

Methods Infants born in Marmara University Hospital were enrolled. Infants of GDMs were assigned as group1 (n:22), LGA infants as group2 (n:17) and the control group was assigned as group3 (n:72). Blood samples for complete blood cell count, ferritin and serum transferrin receptor (sTfR) levels were obtained from cord blood. Blood hemoglobin, ferritin and sTfR levels were compared between the groups.

Results Median head circumference, height and weight of infants born to GDMs and LGA infants were significantly higher than that of the control group. When infants of GDMs and LGA infants were compared, bodyweight and height were significantly higher in the LGAgroup. Nosignificant differences were found in head circumference values between the groups. In groups 1.2 .3 cord blood median hemoglobin, ferritin and sTfRlevels were $17.2 ; 15.4 ; 16.0(\mathrm{gr} / \mathrm{dl})$ $(\mathrm{p}=0.05), 179.7 ; 252.3 ; 225.7(\mu \mathrm{g} / \mathrm{L})(\mathrm{p}=0.456)$ and $5.22 ; 4.34 ; 3.42$ $(\mathrm{mg} / \mathrm{L})(\mathrm{p}=0.008)$ respectively. Hemoglobin levels were higher in the infants of GDMs but this reached only borderline significance $(p=0.05)$. Serum ferritin levels were found to be lower and sTfR levels were found to be higher in the infants of GDMs. The median serum transferrin receptor concentration in the infants of GDMs was significantly higher than that of both the control group and the LGA group. However, the differences between the groups in terms of ferritin were not statistically significant.

Conclusions Increased sTfR reflects tissue iron deficiency in children. Increased sTfR levels in infants of GDMs may indicate that they have an risk for iron deficiency. Optimal follow up is warranted in infants of GDMs to prevent perinatal iron deficiency and its consequences.

752 THE EFFECT OF CHEMOTHERAPY OF NEPHROBLASTOMA A TREATMENT

doi:10.1136/archdischild-2012-302724.0752 